\title{
Ph-ähnlicher Subtyp überraschend häufig
}

Eine Philadelphia-Chromosom-ähnliche (Ph-ähnliche) akute lymphatische Leukämie (ALL) ist ein Subtyp der ALL bei Erwachsenen, der mit einer sehr ungünstigen Prognose einhergeht. Einer aktuellen Untersuchung zufolge kommt der Subtyp sehr viel häufiger vor als erwartet.

Unter 798 ALL-Patienten im Alter zwischen 21 und 86 Jahren hatten Forscher um Kathryn G. Roberts insgesamt mehr als $20 \%$ mit einer Ph-ähnlichen ALL identifiziert: unter den 21- bis 39-Jährigen waren es $27,9 \%$, unter den 40 - bis 59-Jährigen $20,4 \%$ und unter den 60 - bis 86-Jährigen $24,0 \%$.

Patienten mit Ph-ähnlicher ALL ( $\mathrm{n}=155)$ überlebten seltener fünf Jahre krankheitsfrei als Patienten ohne diesen Subtyp ( $\mathrm{n}=247)$ : Der Anteil der Patienten lag bei $22,5 \%$ im Vergleich zu 49,3\% $(\mathrm{p}=0,001)$.
Bei $88 \%$ der Patienten mit Ph-ähnlicher ALL wurden kinaseaktivierende Alterationen gefunden, darunter CRLF2Rearrangements (51\%), ABL-ClassFusionen (9,8\%), JAK2- oder EPORRearrangements (12,4\%), andere JAK-STAT-Sequenzmutationen (7,2\%), andere Kinasealterationen $(4,1 \%)$ und Ras-Pathway-Mutationen (3,6\%). Elf neue Kinase-Rearrangements wurden identifiziert, darunter vier, die neue Kinase- oder Zytokinrezeptorgene betrafen, sowie sieben, die neue Partner für bereits bekannte Gene involvierten.

Die große Varianz der kinaseaktivierenden Alterationen, die bei der Ph-ähnlichen ALL auftreten, hat auch therapeutische Implikationen. Denn bei der Phähnlichen ALL liegt zwar nicht das für das Philadelphia-Chromosom (Ph) übliche BCR-ABL1-Fusionsprotein vor, aber
Mehr als $20 \%$ der erwachsenen ALLPatienten haben eine Ph-ähnliche ALL.

die vorhandenen Rearrangements und Sequenzmutationen deregulieren den Signalweg von Tyrosinkinasen. Deshalb gelten Tyrosinkinaseinhibitoren als eine mögliche Behandlungsoption für Patienten mit Ph-ähnlicher ALL. Studien zur Kombination von konventionellen Therapien mit Tyrosinkinaseinhibitoren sind daher nötig, um die klinische Nutzbarkeit dieser Agenzien bei der Behandlung der Ph-ähnlichen ALL im Erwachsenenalter zu bewerten.

Christina Berndt

Roberts KG et al. High Frequency and Poor Outcome of Philadelphia Chromosome-Like Acute Lymphoblastic Leukemia in Adults. J Clin Oncol. 2017;35(4):394-401.

\section{kurz notiert}

\section{Biosensor detektiert Tumoren} bereits im Frühstadium

Bereits vor der Entwicklung eines Tumors versucht das Immunsystem, gegen veränderte Proteine zu kämpfen, indem es Antikörper gegen diese Proteine einsetzt. Die Bildung solcher Autoantikörper startet oft bereits einige Monate oder sogar Jahre bevor sich der Tumor vollständig entwickelt hat, Symptome entstehen und die Erkrankung von Ärzten erkannt werden kann. Wissenschaftler aus Madrid haben nun einen Biosensor entwickelt, der Antikörper gegen das Protein p53 in Serumproben von Patienten mit kolorektalem Karzinom oder Ovarialkarzinom detektieren kann. P53 repariert DNA-Mutationen, um Veränderungen im Zellzyklus und die Entwicklung von Tumoren zu verhindern. Ist p53 mutiert, produziert der Organismus Autoantikörper dagegen; bei Krebspatienten in 10-40\% der Fälle. Der Nachweis von Autoantikörpern gegen p53 könnte damit auf eine bereits vorhandene oder künftige neoplastische Erkrankung hinweisen. Im Vergleich zu herkömmlichen Methoden bietet der Biosensor, bei dem magnetische Biocarrier verwendet werden, eine 440-mal höhere Sensitivität und eine bessere Unterscheidung zwischen positiven und negativen Serumproben [Garranzo-Asensio M et al. Anal Chem. 2016; 88(24):12339-45].

Judith Neumaier

\section{Prognose beim myelodysplastischen Syndrom}

\section{Faktor Zeit stärker gewichten}

Bei Patienten mit myelodysplastischem Syndrom (MDS), die bei Diagnosestellung einer Hochrisikogruppe zugeordnet werden, kann sich das Risiko vor allem bei jüngeren Erkrankten im Verlauf der Zeit wieder reduzieren. Bei Niedrigrisikopatienten und Älteren bleibt der Risikoscore dagegen auch langfristig relativ konstant. $\mathrm{Zu}$ diesem Ergebnis kam die International Working Group for Prognosis in MDS (IWG-PM) in einer aktuellen Studie. Basis für die Auswertung bildeten die Daten von 7.212 bis dahin nicht behandelten MDS-Patienten. Die Forscher erfassten etablierte Score-Komponenten wie Hämoglobin, Neutrophilen- und Plättchenzahl sowie zytogenetische Merkmale und verfolgten das Risiko für Mortalität und leukämische Transformation mithilfe von Cox-Modellen zu verschiedenen Zeitpunkten nach der Erstdiagnose.

Insgesamt zeigte sich ein Score-Rückgang bei den Risikoparametern für Mortalität bzw. eine myeloische Transformation nach der Diagnose für die Subgruppe der Hochrisikopatienten. Bei der Subgruppe der Niedrigrisikopatienten blieb das Risiko im Verlauf der Zeit dagegen stabil. Nach 3,5 Jahren glichen sich Krankheitsund Transformationsrisiko in den verschiedenen Risikopopulationen an und waren auch noch fünf Jahre nach der Erstdiagnose vergleichbar. Der Verlust der prognostischen Aussagekraft wurde vor allem beim Überleben deutlich. Allerdings führte die Berücksichtigung des Parameters Alter zu einer besseren Prognosestärke schon bei Erstdiagnose.

Um mit dem etablierten IPSS-R ( Revised International Prognostic Scoring System) weiterhin klinisch zuverlässig arbeiten zu können, schlagen die Wissenschaftler einen Cut-off-Wert von 3,5 Punkten für die zeitabhängige Unterscheidung von Hoch- und Niedrigrisikopatienten vor.

Barbara Kreutzkamp

Pfeilstöcker M et al. Time-dependent changes in mortality and transformation risk in MDS. Blood. 2016;128(7):902-10. 